\title{
Enhancing Agile Team Collaboration Through the Use of Large Digital Multi-touch Cardwalls
}

\author{
Martin Kropp ${ }^{1(凶)}$, Craig Anslow ${ }^{2}$, Magdalena Mateescu ${ }^{1}$, Roger Burkhard ${ }^{1}$, \\ Dario Vischi ${ }^{1}$, and Carmen Zahn ${ }^{1}$ \\ 1 University of Applied Sciences Northwestern Switzerland, Windisch, Switzerland \\ \{martin.kropp, magdalena.mateescu\}@fhnw.ch \\ 2 School of Engineering and Computer Science, \\ Victoria University of Wellington, Wellington, New Zealand \\ craig@ecs.vuw.ac.nz
}

\begin{abstract}
Agile software development has become mainstream, and with it many tools have been developed to support Agile software development. Nonetheless, studies show, that most Agile software teams still also use physical cardboards for their daily work. This is error prone and causes a lot of extra effort to keep both in sync. In our research project we conducted an interview study about the reasons for this media break. Based on the findings we developed visualization and interaction concepts for an Agile cardwall using an extra-large multi-touch wall display which provides Agile teams the lightweight collaboration workspace for their Agile meetings. We implemented the concepts in the software prototype $a W a l l$, and evaluated the usability of aWall in a user study. The evaluation indicates that aWall enables and encourages team work due to the large size of the wall, the easy accessibility and visibility of the needed information, and the integration with existing issue tracking tools. This suggests that augmenting digital cardwalls with large interactive touch technology and integration with task tracking systems is a useful way to support effective collaborative Agile software development processes.
\end{abstract}

Keywords: Agile software development - Cardwalls $\cdot$ Large wall displays $\cdot$ Multi-touch $\cdot$ Tool $\cdot$ Software processes $\cdot$ Collaboration

\section{Introduction}

In Agile software development, physical cardwalls continue to be an essential part of the Agile processes despite the relative large number of available digital tools. Although many commercial and open source digital Agile tools like JIRA [3], CA Agile Central (formerly Rally) [16] and VersionOne [12] are available and have been adopted by a large number of Agile companies, studies show that physical cardwalls are still widely used $[4,7]$. Azizyan et al. conducted interviews with software practitioners and found that $31 \%$ of companies used both project management tools and physical cardwalls, where the usage of cardwalls was not restricted to co-located teams [4]. Despite their prevalence, physical cardwalls

(C) The Author(s) 2017

H. Baumeister et al. (Eds.): XP 2017, LNBIP 283, pp. 119-134, 2017.

DOI: $10.1007 / 978-3-319-57633-6 \_8$ 
still have issues as content is not digitalized and not integrated with issue tracking systems. To address the issue with physical cardwalls, we aim to bridge the gap by creating a large digital cardwall that supports elements of the physical nature, integration with existing tracking systems, while also preserving the Agile collaborative work style.

In this paper we present aWall, a large digital cardwall, providing a collaborative workspace for Agile teams. While the main focus of aWall is for use by co-located teams, aWall is designed to be used also by distributed teams which is one of the main driver for digital Agile tools (see Fig. 1). aWall has the size of classical physical cardwalls by using large multi-touch high resolution displays and so provides enough space for the whole team to interactively collaborate. We first give an overview of related work, followed by an evaluation of an interview study about the usage of Agile tools in software teams. We then present the design consideration for a large scale Agile cardwall and the user interface design of aWall. The following section presents the evaluation of aWall in a user study which we conducted with software practitioners to evaluate the usability and effectiveness of the chosen approach. The paper concludes with a final summary and outlook for future work.

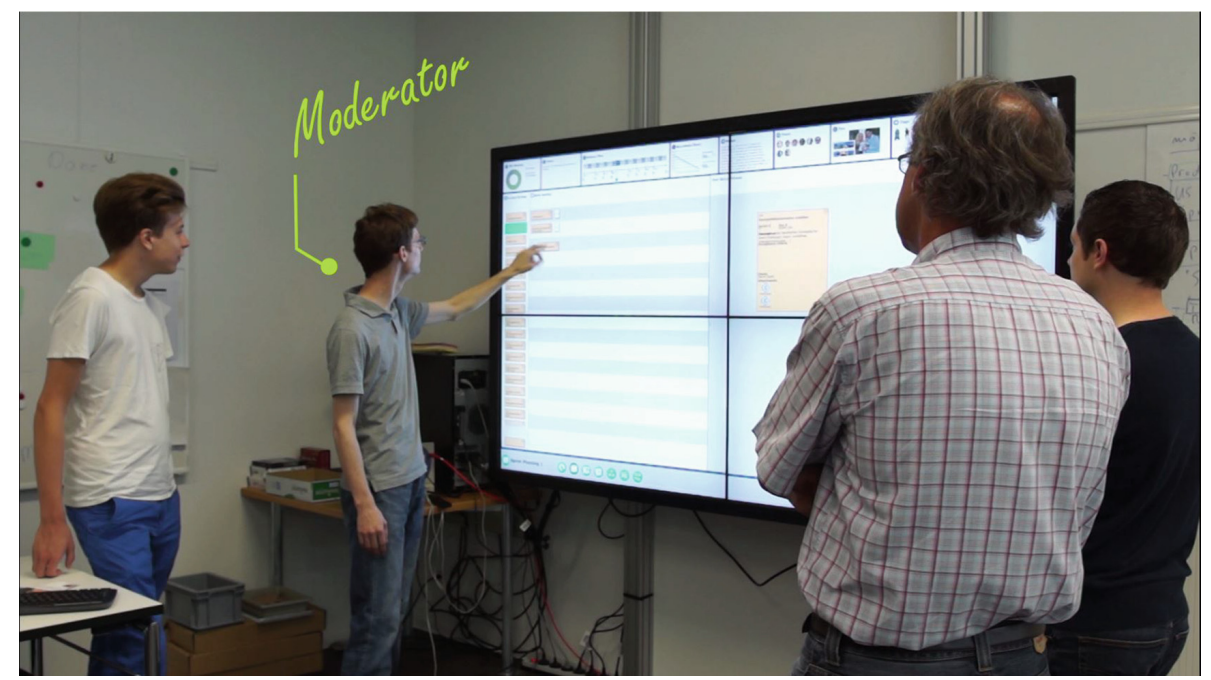

Fig. 1. aWall - digital Agile cardwall displayed on a large high resolution multi-touch wall $(2 \times 246$ Inch $4 \mathrm{~K}$ displays $)$ for planning and Agile team meetings.

\section{Related Work}

Sharp et al. [15] reports that physical artifacts like pin boards, sticky cards, flip charts or whiteboards are used as a means of communication and collaboration by Agile teams. However they also report that there are some disadvantages 
of physical artifacts such as cards may get lost and they cannot be searched or shared easily. Any attempt to overcome these disadvantages by digitalizing cards and cardwalls should retain the advantages of the physical form while also benefiting from translation to the digital medium [15].

Gossage et al. [7] report in their study that the physical nature of artifacts is important to the collaborative process. For example being able to manipulate the cards easily (writing and posting) and their permanent availability on the cardwall helps support effective communication at least in co-located teams. Physical cardwalls are valued for their flexibility, light-weight and easy usage, providing a big picture, and permanent and instant availability of information. Physical cardwalls are not well suited for distributed environments and displaying large amounts of information is difficult. On the other side, they report that digital tools were not necessarily easy to use, hard to personalize, or to adapt to the teams' needs. They come up with suggestions on the design of digital cardwalls and with critical features: always provide an overview, offer easy zoom-and-pan, to hide details, assign annotations to any object on the board, automatic synchronization, for example.

Paredes et al. conducted a survey of existing literature on information visualization techniques used by Agile software development teams and found that information radiators and cardwalls are most frequently used for Agile teams in communication and progress tracking [13].

Azizyan et al. [4] report that digital Agile tools like JIRA and VersionOne account for less than $10 \%$ of tools used to support Agile processes, while physical walls, paper, and spreadsheets account for almost 50\%. They also report that it is important to find the right balance between enough features and usability is critical.

A number of research digital tools have been developed for use on large interactive surfaces (e.g. horizontal and vertical). DAP [10] and subsequently AgilePlanner [18] were early prototypes developed to support Agile planning on horizontal tabletops for co-located teams. SmellTagger supports collaborative code reviews for co-located teams using multi-touch tabletops [11].

CodeSpace [5] does not focus on any particular Agile process but uses shared touch screens, mobile touch devices, and Kinect sensors to share information during developer meetings. They report that professional developers were positive with this approach and felt that pointing with hands or devices and forming hand postures are socially acceptable. Anslow et al. [2] evaluated large display walls for collaborative software visualization. SourceVis used large multi-touch tabletops to support code reviews using collaborative visualization techniques [1]. They show in their paper that large displays have the potential to provide a good overview about complex situations and thus can help to get a better understanding of it. Rubart developed a basic prototype for multi-touch tabletops to support Scrum meetings [14] and evaluated the prototype in a study with student groups. They report positive feedbacks with respect to team collaboration, but also difficulties with editing data. Though support of distributed teams is currently not in the focus of our work, dBoard [6] is a very interesting approach 
in which a Scrum board on a vertical touch screen has been enabled with inscreen video capabilities for distributed development. This might be a possible approach for aWall to support distributed teams.

Based on our review we conclude that most digital Agile tools only partially support collaborative Agile processes and meetings. Digital Agile tools especially seems to lack support for social interaction and team cognitive activities compared with physical tools. Neither existing physical nor digital cardwall tools seem to sufficiently support the collaborative Agile process for Agile teams effectively. With aWall we present an approach to overcome these limitations. aWall tries to combine the advantages of physical and digital cardwalls, by making use of the large screen size and the touch functionality, and serve as an Agile collaborative workspace and information radiator for Agile teams.

\section{Pre-study Tool Usage}

\subsection{Study Method}

As a first step in our project we conducted a qualitative interview study among eleven IT companies that have adopted agile methods in their software development. The interviews focused on collaborative processes in agile teams and the tools they use for communication.

We conducted ten semi-structured group interviews and three individual interviews with eleven IT companies. The interviews were conducted with a total of 44 participants ( 7 female, mean age of all participants was 38.5 years). The participants mostly worked in multiple Agile teams and had different roles in those teams (e.g. Scrum Master, Product Owner, Team Member). The participants had at least one year experience with Agile software development, most between 2-5 years. Each group interview took about two hours and the individual interviews one hour. All interviews were conducted in German. The focus of the interviews was on the employment of agile methods and practices, team and collaborative processes, meetings and tools used. All interviews were audio recorded and transcribed. The transcribed interviews were segmented into small units of analysis and coded using MAXQDA [17]. A category system for the analysis was developed and continuously refined [9].

\section{$3.2 \quad$ Findings}

We found that 10 out of 11 teams still use physical cardwalls typically in combination with digital tools, like Jira/Confluence, TFS, Trello. Only one team was working exclusively with digital tools. When using physical cardwalls we found that it is a common practice to put a lot of extra information around the task board as shown in Fig. 2. The extra information includes for example, the Definition-of-Done, team members' periods of absence, burn-down charts, but also private pictures, post cards from holidays or other greeting cards from team members. 


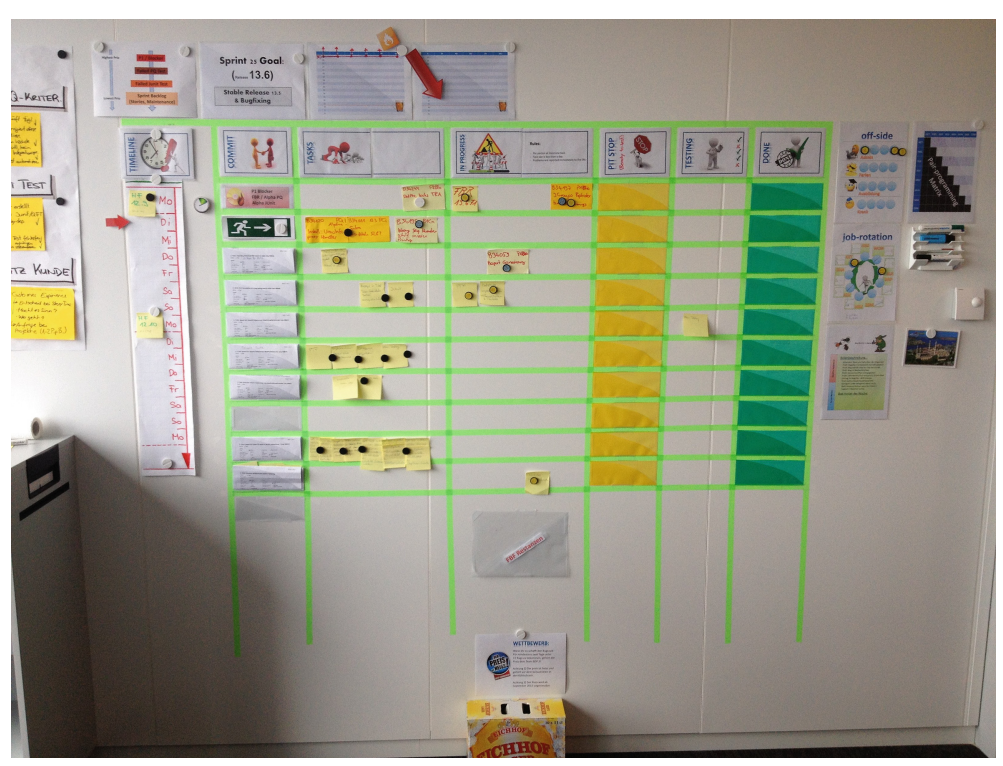

Fig. 2. A physical task board with extra information

When asking for the advantages of physical cardwalls compared to digital Agile boards the most often named reasons were ease of use, always-on visibility, flexibility, good overview, and the focused view on the information. On the disadvantage side the interviewees mainly named that the board is only locally available, the missing traceability and documentation, and the missing integration into digital tools. Table 1 lists the most often named advantages and disadvantages of digital tools by the participants.

The following statements restates opinions from some participants about various aspects about digital tools ${ }^{1}$ :

"Yes, it is all well integrated. If I have it electronically, I have it in the database. I have to capture it only once. That's what I like about the digital tools $(\mathrm{I} 8,305)^{2}$."

"The digital board looks quite nice, is always well ordered (I11, 364)."

"You have so many options in JIRA, so many input fields. And if you are looking for information, you always have to do a lot of navigation.(I1, 235)."

"It takes so long to start up the tool. And after five seconds passed, I have to fill out this template. And then I just want to add a picture. I have to take a photo and somehow add it to the system. That's very cumbersome. (I10, 396)."

\footnotetext{
${ }^{1}$ All statements have been translated from German.

${ }^{2}$ The numbers refer to the interview and the line number of the transcription.
} 
Table 1. Pros and Cons of digital agile tools compared to physical tools

\begin{tabular}{l|l}
\hline Pros & Cons \\
\hline Changes are stored automatically & Feeling of having no control \\
\hline A lot of extra features & Too many features \\
\hline Traceability & Missing visibility for others \\
\hline Transparency in who did what & High effort for usage/administration \\
\hline Provide some overview & Missing good overview \\
\hline Adaptability of tools & High effort for customization \\
\hline Access from everywhere & Not always on (have to start up) \\
\hline Teams can meet in virtual rooms & Must be customized before usage \\
\hline- & Have to navigate to information \\
\hline- & Performance not always good \\
\hline- & Displays are too small \\
\hline
\end{tabular}

We also asked the participants about the requirements for an ideal digital Agile cardwall. The interviewees stressed the importance of non-functional requirements. These included the need for a large size display, configurable views, instant availability of information, overview of information, at all time visible information, easy to reach context dependent information, increased readability of information, simultaneous multi-user touch interaction, direct interaction with data, and no need for navigation.

\subsection{Summary}

In summary the study results seem to show that available digital tools do not sufficiently well support the required flexible collaborative Agile workstyle. Users value the traceability of information in digital tools, linking possibilities of artifacts, and the flexibility to adapt the tools to the users' needs. The main disadvantages of digital cardwall tools seem to be that they are often too complicated to use, the need to navigate to extra information not shown on the main board, no direct and concurrent interaction by all team members, and small displays, missing overview, missing instant availability.

\section{4 aWall - Digital Agile Collaboration Wall}

Based on our study we developed aWall to support Agile teams (co-located or distributed) more effectively than existing physical and digital tools. aWall is designed for various Agile team meetings (e.g. daily stand up, sprint planning, and retrospectives) by providing information dashboards, maintaining user stories and tasks, enables customization of Agile processes, and integrates with issue tracking systems. aWall was developed by an interdisciplinary project team of 
computer scientists and psychologists (from the School of Engineering, and the School of Applied Psychology). We now outline the design and user interface of aWall, followed by a user study to evaluate the prototype.

\subsection{Design}

Based on the requirements elicited during the interviews, we identified a number of design considerations.

Physical Size. A digital cardwall needs to satisfy not only the needs for interacting with the digital content, but also provide enough physical space to display information to effectively support team collaboration. Therefore, the size of a digital cardwall needs to be at least comparable to that of physical cardwalls. aWall consists of four $46 \mathrm{in}$. displays $(2 \times 2)$, for a wall size of $2.05 \mathrm{~m}$ width and $1.25 \mathrm{~m}$ height (see Fig. 1).

High Resolution. Each display in aWall is $3840 \times 2160$ pixels, for a total resolution of $15360 \times 8640$ pixels. The high resolution display wall provides enough real estate to display large amounts of information at once while still ensuring the readability of text elements, widgets, and views.

Multi-user and Multi-Touch. The display wall consists of a 12 point multi-touch infrared optical overlay (PQ Labs frame ${ }^{3}$ ) which is attached to the display wall. The multi-touch capabilities allows multiple users to work simultaneously with artifacts and provides an accurate and effective touch experience.

Integration with Issue Tracking Systems. aWall is designed to run on top of existing third party issue tracking systems such as JIRA. Therefore, infrastructure functionality can be reused and already defined Agile processes utilized.

Availability of Information. aWall can replace physical cardwalls and act as the team's external memory of the project. For that, aWall should be installed in a team's open office area, always being switched on, and have a permanent view of the task board.

Web Technologies. In order to have a ubiquitous and easily deployable design, aWall was developed as a web application based on HTML5 and JavaScript technology. For multi-touch support we used the interact.js framework ${ }^{4}$.

\subsection{User Interface}

The aWall user interface contains a number of different views, widgets, and interaction techniques designed to support different types of Agile meetings.

Action and Information View. The results of the interviews showed that most interaction with the cardwall takes place during Agile meetings. Each meeting

\footnotetext{
${ }^{3}$ http://multitouch.com/.

${ }^{4}$ http://interactjs.io/.
} 
has specific goals, operates on different data, and requires different supporting tools and information. To support these different types of information handling, we divide the display into an action view and an information view. Figure 3 shows the view for a daily standup meeting highlighting the separation into information view and action view. The action view is the main work area, which is dedicated to the core artifacts of a specific meeting. The main interactions during a meeting are performed by users on the action view. The information view provides supporting information and tools needed for the meeting. The information view represents the dynamic memory of the team and as any dynamic system they need to allow for change. For example, the information view for the daily standup meeting contains additional information, like a timer widget showing the meeting moderator and a countdown, a team widget showing the team members, a definition-of-done widget, an impediment list widget, and a burn-down chart for an iteration. When necessary, new widgets can be added and removed from the information view.

Dedicated Views. aWall provides dedicated views that are tailored to the specific needs of Agile meetings. For the sprint planning meeting shown in Fig. 4, the action view is divided into three columns. The left column shows the top priority user stories of the product backlog. The centre column shows the so far selected user stories for the next iteration. The right column shows a detailed view of the currently selected user story. This column can be used by the product owner to discuss and clarify open issues during the meeting with the development team. Relevant documents can be easily attached and opened in the application. Figure 5 shows the retrospective meeting view after team members have sent their iteration feedback where the notes have been ordered on the right side. Users can navigate between the different meeting views by means of a navigation bar displayed at the bottom of the view.

Information Widgets. The information view consists of a set of widgets (e.g. team widget, timer widget, fun widget, avatar widget - see Figs. 3, 4, 5) and can be independently configured for each Agile meeting. Each widget is designed to support distinct aspects of the collaborative Agile process. The team widget shows the team members and can be used to assign people to tasks during a daily standup meeting. The timer widget supports time boxing during the meeting and furthermore, allows to choose a meeting moderator. The moderators' names are stored in the application and future moderators can be suggested based on previous selections. The fun widget allows users to post personal or fun images to the information view to help bring emotion to the cardwall and foster team thinking. The avatar widget can be used to drag avatars to any position on the wall or attach it to tasks or user stories. Both the fun and avatar widgets are designed to help with the interpersonal process in Agile teams (emotion management, team spirit). All widgets can be detached from the information view and moved around the cardwall to facilitate user interaction.

Availability of Information. Any information needed for a meeting is visible and accessible; either on the action view or on the information view. If the team 


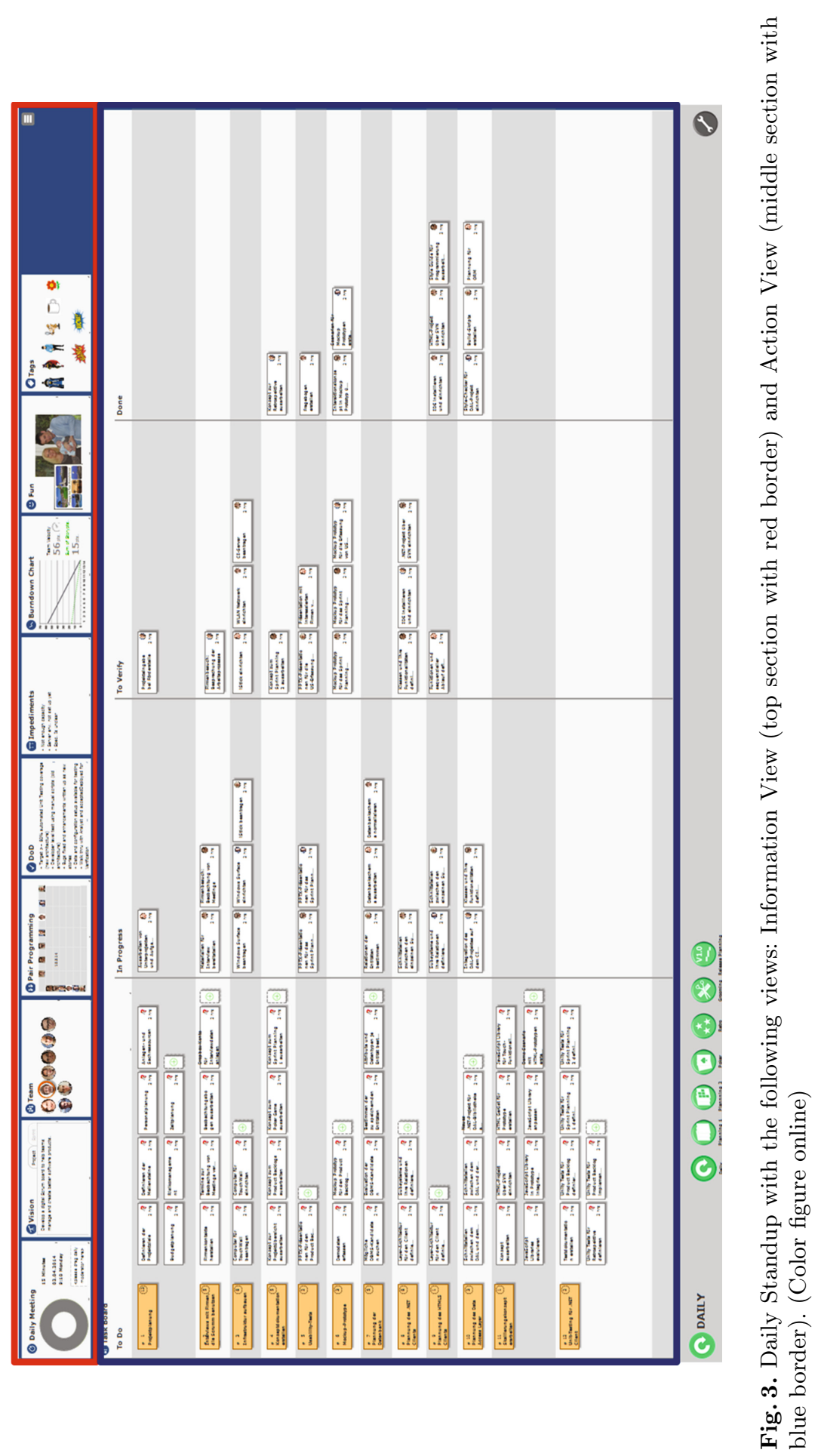




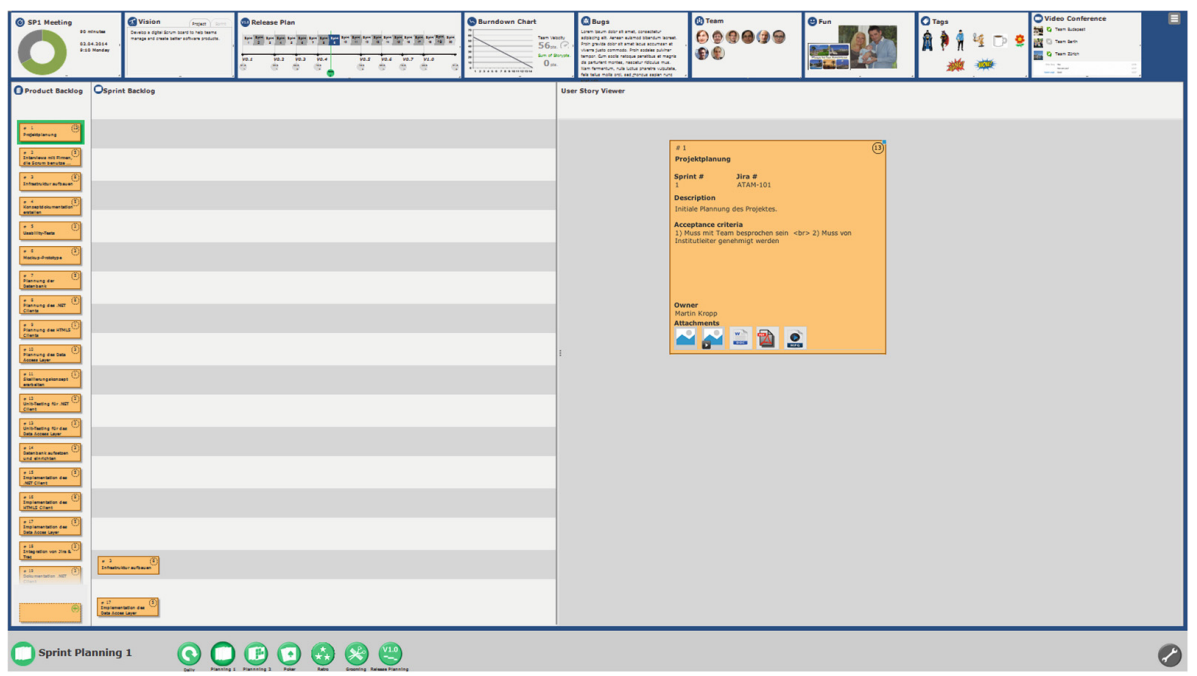

Fig. 4. Sprint planning meeting with a user story detail view.

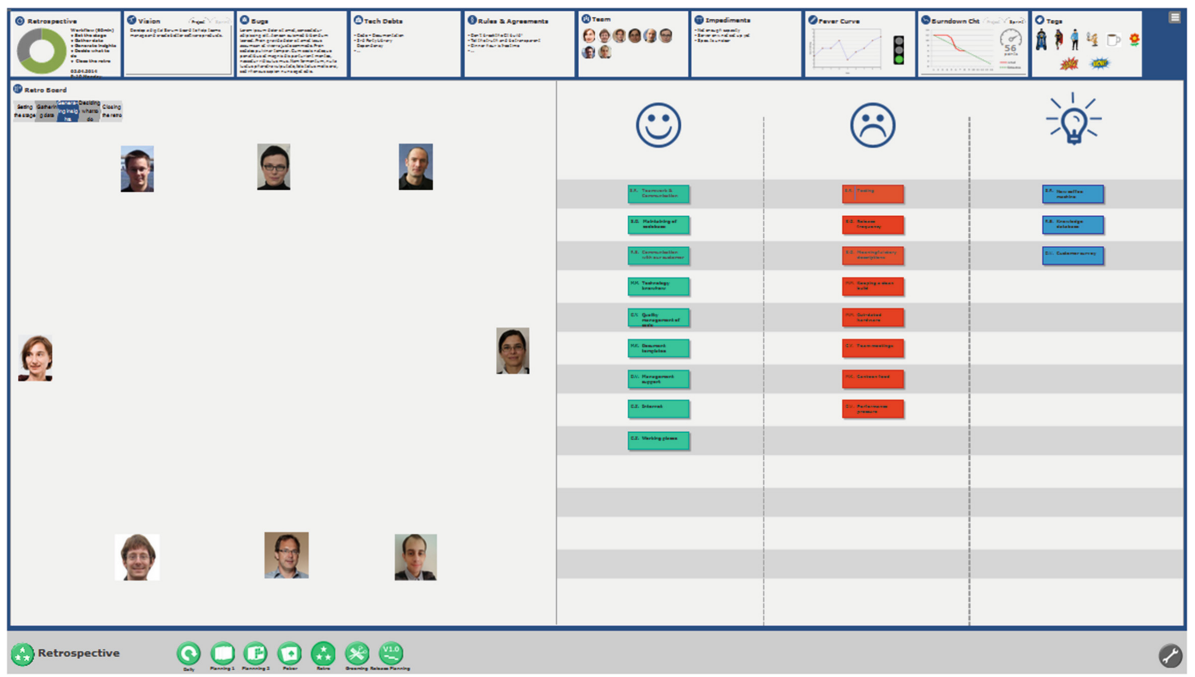

Fig. 5. Retrospective meeting view.

needs different supporting information, additional widgets can be switched on or off in the configuration button on the right side of the information view.

Interaction. aWall supports multi-touch and multi-user interaction. Fluid interaction with widgets and cards is enabled by gestures like tap, double tap, dragand-drop, and pinch-to-zoom supporting changing task and user story cards position, moving widgets around the cardwall, and changing the size of a widget. 
Data can be either entered on the cardwall with a virtual or physical keyboard or via the underlying issue tracker system and mobile devices such as tablets.

Scalability of Information. By default, user story cards and task cards show only a few details (e.g. title). By increasing the card size with a pinch-to-zoom gesture more information is displayed. The text size increases concomitantly with the widening of the cards so that information can be more easily read depending on the distance from the cardwall. When all information is shown the widget automatically switches into edit mode, so that data can be added or modified.

\section{$5 \quad$ User Study}

To evaluate the usability of the aWall prototype we conducted a qualitative user study with professional Agile practitioners. The goals were:

1. evaluate the availability of context specific information

2. evaluate reachability and discoverability of functionality and information

3. evaluate the support of Agile workstyle and Agile culture,

4. evaluate the applicability to real life situations in Agile teams.

The user study was conducted with an early prototype of aWall. The participants worked in teams and had to complete various tasks.

\subsection{Participants}

We recruited 11 employees (nine men and two women - see Table 2) from the same companies that participated in our interview study [8]. Most participants had many years of experience in IT, and several of them in Agile development. They came from different fields and covered a wide spectrum of Agile team roles. Among the participants were four Scrum Masters, two Agile coaches, two senior developers, one Agile grandmaster, one UX consultant and one head of a software development department. Two of the companies were from the assurance domain, one manufacturing, two service providers, one engineering, and one enterprise software development company. Four companies sent two employees, and three companies sent one employee each. All companies had been applying Agile processes for at least one year, and all employees to executed the given tasks in their companies before.

\subsection{Procedure}

We divided the eleven participants randomly into two groups by five and six people. Both groups completed the same tasks with aWall in two separate workshop sessions. Each workshop session lasted one hour.

Upon signing an informed consent statement, the participants were asked to act as a team during the workshop. Prior to the user study, the participants received a presentation on the interview study results, but did not receive any 
Table 2. Demographics of workshop participants: gender, IT experience, Agile experience, job title, company (anonymized), and workshop group.

\begin{tabular}{l|l|l|l|l|l}
\hline Gender & IT Exp. & Agile Exp. & Job title & Company & Group \\
\hline Male & 23 & 3 & Head SW dev & D & 1 \\
\hline Male & 5 & 1.5 & Senior dev & E & 1 \\
\hline Male & 13 & 2 & Grandmaster & C & 1 \\
\hline Male & 10 & 3 & Agile coach & F & 1 \\
\hline Male & 19 & 4 & Senior dev & G & 1 \\
\hline Male & 10 & 3 & UX consultant & B & 1 \\
\hline Female & 8 & 3 & Agile coach & C & 2 \\
\hline Female & 15 & 5 & Scrum master & A & 2 \\
\hline Male & 15 & 3 & Scrum master & A & 2 \\
\hline Male & 1 & 1 & Scrum master & E & 2 \\
\hline Male & 6 & 2 & Scrum master & F & 2 \\
\hline
\end{tabular}

information about the aWall application. Each member of the team received three tasks to be solved together in groups using aWall. The tasks involved a daily standup meeting and a sprint planning meeting. After receiving the task, each participant read the task out aloud to the other participants and completed it with their help.

The daily standup tasks included to start the daily standup meeting (task 1), choose a moderator for the meeting (task 2), and update the task board during the meeting (task3), assign team members to a task card (task 4). For example: "In this team you play the role of team member $M$. Please find a way to carry out a daily standup. The application suggests a moderator. Please ask the team member suggested by the application to play the moderator. Please act as a team accordingly to the received instructions.". The sprint planning tasks included to show and discuss a user story during the meeting (task 1) and move the story to the sprint backlog (task 2). Other tasks were to switch on and off different widgets in the information view.

After completing the tasks for each type of meeting we asked the participants about the benefits and difficulties of aWall in an open discussion. The discussions were recorded and the results written down. Both team workshops were conducted by two moderators.

\subsection{Findings}

The overall feedback for the prototype was very positive, with the participants considering aWall to be usable, capable to support Agile processes in general and especially the collaborative working style in teams.

Size Aspects. The participants especially valued the large size and high resolution of aWall. The large size supports real team collaboration capabilities, similar to 
physical cardwalls. Displaying a large amount of information at once was deemed positive. As one participant stated ${ }^{5}$ :

"With the large size you can display many user stories and tasks."

Readability of Information. Most participants considered the displayed information to be legible, especially since the card titles are relatively large. Some participants considered the actual cards to be too small. Therefore, it is very important to be able to display the whole content of a card and enlarge the font size so that the whole team can read it from a distance. One participant stated:

"That's really a nice feature, that cards can be enlarged and font size increases to improve readability."

Availability of Information. The participants especially valued the availability of additional information and functionality for the different meetings. The separation of the display into action view and information view was easily understood. Some participants mentioned that elements placed on the upper side of the display wall might be out of reach for smaller people. Another participant liked the extra features:

"I like the extra features around the main view and the additional information."

Discoverability of Functionality. The participants discovered most functionality of aWall by themselves and could easily interact with the display wall. There were some issues with discoverability of those functions that were not a straight-forward transfer of the pin-boards into the digital world. For example, the timer widget has no corresponding artifact in the practice of Agile teams. Whereas, direct implementations of the pin-boards functionality (e.g. the task-board shown in the daily standup meeting) were instantly understood and deemed as valuable by the participants. That was also the case for the widgets inspired from Agile practices such as the team widget which is based on the observation that Agile teams sometimes write the team members' names on the cards or even hang their pictures on the pin-boards.

Third-Party System Integration. The integration with third-party tools was positively rated. Tasks modified during the daily standup meeting, are immediately synchronized in the Agile project management tool (JIRA). There is no extra effort to update the tasks manually from the physical cardwall after the meeting. One participant stated:

"The link to JIRA with automatic update of data is important."

Flexibility and Customization. Increased flexibility with respect to both the manner of conducting the meetings and displaying information was considered important by the participants. For example, the timer widget solicited choosing a moderator at the beginning of a meeting. The flexibility provided by aWall was also

\footnotetext{
${ }^{5}$ All quotes have been translated from German.
} 
positively rated, especially with respect to conducting retrospective meetings that sometimes might prove strenuous. The participants considered that it is important to create a proper environment especially for this type of meeting as sometimes they tend to transmute into a drill. Most participants were in favour of a greater flexibility of the time boxing, with only optionally choosing a moderator and not showing the elapsed time, but the time of day during the daily meeting. The participants valued the team widget, but requested to have more information being displayed (e.g. absences, vacation days) and allow for more customization. Furthermore, the participants remarked that they should be able to add functionality to aWall on their own and not be dependent on standard functionality as often is the case with other Agile tools.

Agile Collaborative Workspace. Offering tags and avatars as well as the fun view was positively seen as bringing emotions onto the board. One participant mentioned the positive effect of avoiding media disruption, by being able to do all interaction with only one medium:

"With such a board we could probably avoid media discontinuity."

Filtering and Representation of Information. The participants requested especially to have filter functions, to highlight and show the desired information. As an example, participants requested to highlight all tasks of a team member, when touching that person in the team view. The usage of colors for different types of user stories was suggested to increase readability (e.g. to distinguish between technical tasks, bug reports, or user requirements).

Task Time Recording. Some participants suggested automatically capturing the time spent on a task combined with computing of the work hours on the task would help provide further metric details of performance.

Provenance of Information. Some participants suggested to have automatic recordings of meetings with voice recognition and transcriptions of the discussions form the interactions in front of the display wall for later recollection and analysis of the meetings.

\section{Conclusions}

Current Agile cardwalls don't fulfil today's requirements for effective software development. We aim to bridge that gap with aWall, a digital cardwall tool to support co-located and distributed Agile teams. aWall provides a collaborative workspace using large multi-touch displays, information transparency, direct information interaction without the need for navigation, support for the whole Agile process, and dedicated views for different types meetings. We conducted a user study with 11 Agile practitioners and found that they especially valued the large-size of the wall due to the physical space affordances, the dedicated views with context specific information, and the always visible and direct information access. Our future work involves deploying aWall within companies. 
Acknowledgments. Many thanks goes to the University of Applied Sciences Northwestern Switzerland for funding this project as part of their strategic initiative to fostering interdisciplinary work, and to the companies and people for participating in this project. Thanks to Robert Biddle for feedback on early drafts of this paper.

\section{References}

1. Anslow, C., Marshall, S., Noble, J., Biddle, R.: Sourcevis: collaborative software visualization for co-located environments. In VISSOFT, pp. 1-10. IEEE (2013)

2. Anslow, C., Marshall, S., Noble, J., Tempero, E., Biddle, R.: User evaluation of polymetric views using a large visualization wall. In: SoftVis, pp. 25-34. ACM (2010)

3. Atlassian: JIRA (2015). https://www.atlassian.com/software/jira

4. Azizyan, G., Magarian, M.K., Kajko-Matsson, M.: Survey of agile tool usage and needs. In AGILE, pp. 29-30. IEEE (2011)

5. Bragdon, A., DeLine, R., Hinckley, K., Morris, M.: Code space: touch + air gesture hybrid interactions for supporting developer meetings. In: ITS, pp. 212-221. ACM (2011)

6. Esbensen, M., Tell, P., Cholewa, J.B., Pedersen, M.K., Bardram, J.: The dBoard: a digital scrum board for distributed software development. In ITS, pages 161-170. ACM, 2015

7. Gossage, S., Brown, J., Biddle, R.: Understanding digital cardwall usage. In AGILE, pp. 21-30. IEEE (2015)

8. Mateescu, M., Kropp, M., Greiwe, S., Burkhard, R., Vischi, D., Zahn, C.: Erfolgreiche zusammenarbeit in agilen teams: Eine schweizer interview-studie ber kommunikation, 22 December 2015. http://www.swissagilestudy.ch/studies

9. Mayring, P.: Einfhrung in die qualitative Sozialforschung. Beltz-UTB, Weinheim (2003)

10. Morgan, R., Walny, J., Kolenda, H., Ginez, E., Maurer, F.: Using horizontal displays for distributed and collocated agile planning. In: Concas, G., Damiani, E., Scotto, M., Succi, G. (eds.) XP 2007. LNCS, vol. 4536, pp. 38-45. Springer, Heidelberg (2007). doi:10.1007/978-3-540-73101-6_6

11. Muller, M., Wursch, M., Fritz, T., Gall, H.: An approach for collaborative code reviews using multi-touch technology. In: CHASE Workshop. ACM (2012)

12. Version One. Enterprise agile platform. (2015). http://www.versionone.com. Accessed 6 Jan 2017

13. Paredes, J., Anslow, C., Maurer, F.: Information visualization for agile software development teams. In: VISSOFT, pp. 157-166. IEEE (2014)

14. Rubart, J.: A cooperative multitouch scrum task board for synchronous face-to-face collaboration. In: ITS, pp. 387-392. ACM (2014)

15. Sharp, H., Robinson, H., Petre, M.: The role of physical artefacts in agile software development: two complementary perspectives. Interact. Comput. 21(1-2), 108-116 (2009)

16. CA Technologies. CA agile central (2016). https://www.ca.com/. Accessed 6 Jan 2017

17. Research GmbH VERBI Software, Consult. Maxqda data analysis software (2015). http://www.maxqda.com. Accessed 6 Jan 2017

18. Wang, X., Maurer, F.: Tabletop agileplanner: a tabletop-based project planning tool for agile software development teams. In: TABLETOP, pp. 121-128. IEEE (2008) 
Open Access This chapter is licensed under the terms of the Creative Commons Attribution 4.0 International License (http://creativecommons.org/licenses/by/4.0/), which permits use, sharing, adaptation, distribution and reproduction in any medium or format, as long as you give appropriate credit to the original author(s) and the source, provide a link to the Creative Commons license and indicate if changes were made.

The images or other third party material in this chapter are included in the chapter's Creative Commons license, unless indicated otherwise in a credit line to the material. If material is not included in the chapter's Creative Commons license and your intended use is not permitted by statutory regulation or exceeds the permitted use, you will need to obtain permission directly from the copyright holder.

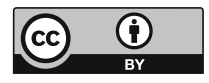

